\title{
DOKUMENTATION
}

\section{Dekret des Ministerrates Nr. 139-HDBT vom 5. September 1988 über die Einzelheiten der Durchführung des Gesetzes über ausländische Investitionen in Vietnam ${ }^{1}$}

\section{Übersicht}

Kapitel I: $\quad$ Allgemeine Bestimmungen

Kapitel II: $\quad$ Vertragliche Unternehmens-Kooperationen

Kapitel III: $\quad$ Joint Venture

Kapitel IV: $\quad$ Privatunternehmen mit $100 \%$ iger ausländischer Kapitaleinlage

Kapitel V: $\quad$ Geschäftsorganisation

Kapitel VI: Arbeitsbeziehungen in Unternehmen mit ausländischem Investitionskapital

Kapitel VII: $\quad$ Finanzangelegenheiten

Kapitel VIII: Devisenkontrolle

Kapitel IX: $\quad$ Buchführung und Rechnungsprüfung

Kapitel X: Zölle, Einreise, Aufenthalt und Verkehr

Kapitel XI: Schlußbestimmungen

Im Hinblick auf das Gesetz über die Organisation des Ministerrates der Sozialistischen Republik Vietnam (SR Vietman) vom 4. Juli 1981, in Ubereinstimmung mit dem Gesetz über ausländische Investitionen in Vietnam vom 29. Dezember 1987 und auf Vorschlag des Ministers für Außenwirtschaftsbeziehungen (MfAW) erläßt der Ministerrat folgendes Dekret:

1 Quelle: ICSID Review - Foreign Investment Law Journal 4 (1989), 406-433 = Bundesstelle für Außenhandelsinformation - Dokument Nr. R 107/90 v. 5.11.1990, 11-32. 


\section{Kapitel I: Allgemeine Bestimmungen}

Art. 1: Das vorliegende Dekret konkretisiert das Gesetz über ausländische Investitionen in Vietnam (im folgenden kurz Investitionsgesetz) mit dem Ziel, günstige Bedingungen für dessen ordnungsgemäße Durchfühnung zu schaffen.

Art. 2: Die Begriffsbestimmungen des Artikels 2 des Kapitels I des Investitionsgesetzes gelten auch für die in diesem Dekret verwendeten Begriffe. Begriffe, für die im Investitionsgesetz keine Begriffsbestimmung enthalten ist, werden in den einschlägigen Vorschriften dieses Dekrets bestimmt.

Art. 3: Dieses Dekret regelt die Einzelheiten der in Kapitel II des Investitionsgesetzes vorgesehenen Formen direkter Investitionstätigkeiten von Ausländern in Vietnam. Indirekte Investitionstätigkeiten, wie Kapitalkredite usw., fallen nicht unter den Geltungsbereich dieses Dekrets.

Art. 4: Das Investitionsgesetz regelt folgende Gegenstände:

1. Vietnamesische Wirtschaftseinheiten mit dem Status einer juristischen Person einschließlich Einzelunternehmen, Unternehmenskomplexe, Unternehmensverbindungen und sonstige staatliche Wirtschaftseinheiten, gemischt staatlich-private Gesellschaften und Produktionsgenossenschaften.

Organe der Wirtschaftsverwaltung können keine Investitionskooperationen mit ausländischen Partnern eingehen.

2. Vietnamesische Privatpersonen einschließlich privater Gesellschaften dürfen in die oben erwähnten vietnamesischen Wirtschaftsorganisationen, welche als vietnamesische Partner von Untemehmens-Kooperationen mit einem ausländischen Partner auftreten, Kapital einbringen.

3. Auslandsvietnamesen, welche Direktinvestitionen in Vietnam vomehmen, haben unabhängig von ihrer Nationalität dieselben Rechte und Pflichten wie ausländische Investoren. Bringen sie in eine der in Absatz 1 dieses Artikels genannten vietnamesischen Wirtschaftsorganisationen oder mehrere davon Kapital ein, damit diese als vietnamesischer Partner einer Unternehmens-Kooperation mit einem ausländischen Partner auftreten können, so haben sie die gleichen Rechte und Pflichten wie eine vietnamesische Privatperson. In beiden Fällen werden ihnen gesondert zu regelnde Vergünstigungen zuteil.

4. Unternehmen mit ausländischem Investitionskapital mit dem Status einer juristischen Person. 
5. Ausländische Wirtschaftsorganisationen mit dem Status einer juristischen Person einschließlich nationaler und internationaler Wirtschafts- und Finanzeinrichtungen und ausländische Privatpersonen, welche Direktinvestitionen in Vietnam vornehmen.

Art. 5: Die in Artikel 4 genannten Wirtschaftsorganisationen mit dem Status juristischer Personen haben folgende Voraussetzungen zu erfüllen:

(1) Sie müssen in Übereinstimmung mit dem Gesetz errichtet worden sein.

(2) Sie müssen über eigenes Vermögen verfügen und dafür selbständig verantwortlich sein.

(3) Sie müssen das Recht haben, selbständige Entscheidungen über ihre Produktion und Geschäftstätigkeit zu treffen.

(4) Sie müssen die Fähigkeit besitzen, selbständig Zivilrechtsbeziehungen einzugehen, d.h. Verträge abzuschließen und vertragliche Verbindlichkeiten zu erfüllen und als Kläger und Beklagte vor Gericht aufzutreten.

Art. 6: Das in Kapitel V des Investitionsgesetzes genannte Staatsorgan für die Verwaltung ausländischer Investitionen der Regierung der SR Vietnam ist das Staatskomitee für Kooperationen und Investitionen (SKKI).

Das Mf AW ist zuständig für den Empfang und die Bearbeitung von Investitionsprojekten ausländischer Investoren, für die Empfehlung alternativer Bearbeitungsansätze und für die Berichterstattung gegenüber dem SKKI, bei welchem die Prüfung und Entscheidung liegt.

Art. 7: 1. Ausländische Organisationen und Privatpersonen, die in einer der in Artikel 4 des Kapitels II des Investitionsgesetzes vorgesehenen Formen investieren, haben das in diesem Dekret vorgeschriebene Verfahren zu beachten.

2. Ein Ausländer, der in Vietnam zu investieren beabsichtigt und noch keinen geeigneten Partner für eine Kooperation gefunden hat, kann sich zu diesem Zweck an eine zentrale oder regionale Gesellschaft für Investitionsdienste oder das MfAW wenden.

3. Vor dem Aushandeln oder Abschluß eines Kooperationsprojektes mit einem ausländischen Partner hat der vietnamesische Partner eine Machbarkeitsstudie durchzuführen und die Zustimmung der zuständigen Verwaltungsbehörde (je nachdem auf Zentral-, Provinz-, Stadt- oder Sonderzonenebene) einzuholen.

4. Offizielle Gebühren für die Ưberprüfung des Antrages auf Untemehmens-Kooperation oder Investition, für die Erteilung einer Geschäftslizenz, einer Investitionslizenz oder einer Urkunde über die Registrierung der Satzung des Unternehmens, für die Unterrichtung der zuständigen Behörden und die Bekanntmachung im Amtsblatt sind vom Antragsteller nur einmal zur Zeit der Antragstellung zu entrichten. 
In einem Vertrag über Unternehmens-Kooperation oder ein Joint Venture haben die beiden Partner zu vereinbaren, wer von ihnen die Gebühren zu tragen hat.

Art. 8: Die dem SKKI zu übersendenden Dokumente sind in vietnamesischer oder sowohl in vietnamesischer als auch in einer weitverbreiteten fremden Sprache abzufassen. Sowohl der vietnamesische als auch der ausländische Text sind gleichwertig authentisch.

\section{Kapitel II: Vertragliche Unternehmens-Kooperationen}

Art. 9: 1. Ein Unternehmens-Kooperations-Vertrag ist eine Urkunde, die von einem vietnamesischen und einem ausländischen Partner mit dem Ziel unterzeichnet worden ist, gemeinsam eine der oder mehrere Produktions- oder Geschäftstätigkeiten in Vietnam auf der Grundlage der wechselseitigen Zuweisung von Verantwortlichkeiten und der Verteilung der Geschäftsgewinne aufzunehmen, ohne ein Joint-Venture-Unternehmen oder eine sonstige neue juristische Person zu gründen.

Handels- und sonstige Wirtschaftsverträge über den Güteraustausch, wie Lieferung von Rohstoffen im Austausch gegen Endprodukte usw., fallen nicht in den Geltungsbereich dieses Dekrets.

2. Die Laufzeit eines Unternehmens-Kooperations-Vertrages ist zwischen den Parteien unter Berücksichtigung der Natur des Geschäfts und der für die Erfüllung der Vertragsziele erforderlichen Zeitdauer zu vereinbaren.

Art. 10: Ein Unternehmens-Kooperations-Vertrag ist von den ordnungsgemäß bevollmächtigten Vertretern der Vertragsparteien zu unterzeichnen.

Art. 11: Dem Antrag auf Genehmigung eines Unternehmens-Kooperations-Vertrages und Erteilung einer Geschäftslizenz beim SKKI hat der Antragsteller folgende Dokumente beizufügen:

(a) der zwischen den Parteien vereinbarte Unternehmens-Kooperations-Vertrag;

(b) eine Darstellung der wirtschaftlich-technischen Grundlagen des Vertrages;

(c) die erforderlichen Informationen über die Parteien, wie die Satzung der Gesellschaft oder die Geschäftsfähigkeit der Einzelperson als Partei eines Vertrages und die finanziellen Verhältnisse der Vertragsparteien;

(d) sonstige vom SKKI zur Klärung von Fragen im Zusammenhang mit den oben erwähnten Informationen und Dokumenten angeforderte Unterlagen; und

(e) der Antrag auf Gewährung von Vergünstigungen, soweit vorgesehen. 
Art. 12: Das SKKI hat seine Entscheidung den Partnem einer Unternehmens-Kooperation innerhalb von drei Monaten vom Zeitpunkt des Eingangs des Antrages auf Erteilung einer Geschäftslizenz an mitzuteilen. Wenn der Vertrag genehmigt wird, hat das SKKI den Partnem die Geschäftslizenz zu erteilen, Abschriften der genannten Lizenz an die Zentralbehörde und das Volkskomitee der zuständigen Provinz, Stadt oder Sonderzone zu senden und sie danach im Amtsblatt der Regierung der SR Vietnam bekanntzumachen.

Art. 13: Bei der Überprüfung eines Antrages auf Erteilung einer Geschäftslizenz hat sich das SKKI von der Ordnungsgemäßheit des Untemehmens-Kooperations-Vertrages zu überzeugen.

Der Vertrag hat u.a. folgendes zu enthalten:

(a) Alle erheblichen Daten betreffend die Vertragsparteien, wie ihre Staatsangehörigkeit, ihre Adressen und ihre ordnungsgemäß bevollmächtigten Vertreter;

(b) eine Beschreibung der beabsichtigten Geschäftstätigkeiten;

(c) eine Liste über die Quantität und Qualität der Grundausrüstung und der für die Geschäftstätigkeit notwendigen Materialien und Quellen ihrer Bedarfsdeckung, die Spezifikation, Quantität und Qualität der Produkte und deren Absatzmöglichkeiten; bei Dienstleistungsverträgen ist das Verhältnis von frei vereinbarter konvertibler Währung zur vietnamesischen Währung im Hinblick auf Dienstleistungsentgelte ausdrücklich anzuführen;

(d) die Rechte und Pflichten der Parteien und die Bedingungen der Übertragung der jeweiligen Rechte und Pflichten aus dem Vertrag;

(e) die Klauseln betreffend Laufzeit, Änderung und Beendigung des Vertrages; und

(f) das Verfahren betreffend die Beilegung von Streitigkeiten zwischen den Parteien, die bei Ausführung des Vertrages entstehen.

Art. 14: Ein Unternehmens-Kooperations-Vertrag wird erst wirksam, wenn er vom SKKI genehmigt und zu diesem Zweck eine Geschäftslizenz erteilt worden ist.

Art. 15: Die Übertragung von Rechten und Pflichten aus dem Vertrag durch eine der Parteien auf einen Dritten bedarf der vorherigen Zustimmung der anderen Partei.

Der Dritte, der die Rechte und Pflichten einer Partei aus dem Vertrag übernimmt, hat dem SKKI die in Buchstaben (c) des Artikels 11 dieses Dekrets aufgeführten Dokumente und Informationen zu übersenden.

Eine Übertragung der Rechte und Pflichten einer der Parteien aus dem Vertrag ist nur gültig, wenn sie vom SKKI genehmigt worden ist. 
Art. 16: Wenn die Parteien übereinkommen, die Laufzeit des Vertrages zu verlängern, so haben sie einen entsprechenden Antrag spätestens einen Monat vor Beendigung des Vertrages beim SKKI zu stellen. Das SKKI hat seine Entscheidung dem Antragsteller schriftlich innerhalb von fünfzehn Tagen vom Zeitpunkt des Eingangs des Antrages an mitzuteilen.

Art. 17: 1. Ein Unternehmens-Kooperations-Vertrag kann vor dem Beendigungszeitpunkt aufgelöst werden, wenn die in dem Vertrag ausbedungenen Voraussetzungen für die Auflösung erfüllt sind.

2. Sind die Voraussetzungen für eine Auflösung nicht erfüllt, bleibt der Vertrag trotz Eintritts des Beendigungszeitpunktes wirksam, es sei denn, die Parteien vereinbaren die Auflösung.

3. Nach Beendigung des Vertrages bleiben die in dem Vertrag ausbedungenen Klauseln über die Beilegung von Streitigkeiten und die Geltendmachung von Ansprüchen bis zum Ablauf der gesetzlichen Verjährungsfrist für Schiedsverfahren und Rechtsstreitigkeiten oder der in Ermangelung einer gesetzlichen Ver jährungsfrist von den Parteien vereinbarten Frist wirksam.

Art. 18: Die Parteien eines Unternehmens-Kooperations-Vertrages haben dem SKKI spätestens bis zum 30. März eines jeden Jahres Bericht über das Ergebnis der Vertragstätigkeit des zurückliegenden Jahres zu erstatten.

Art. 19: Jede Partei eines Untemehmens-Kooperations-Vertrages

(a) sorgt für die Zahlung aller fälligen Steuern;

(b) trägt nach dem Recht der SR Vietnam Verantwortlichkeit für ihre eigenen Handlungen.

\section{Kapitel III: Joint Ventures}

Art. 20: In diesem Kapitel werden folgende Begriffe in der nachstehenden Bedeutung gebraucht:

1. "Gesamtinvestitionskapital eines Joint Venture" bedeutet die Summe aller Kapitalgegenstände zur Ausführung eines Joint-Venture-Projekts, das sowohl das Anlage- als auch das Betriebskapital, einschließlich der Kredite umfaßt, die notwendig für die Tätigkeit des Joint Venture sind. 
2. "Gesetzliches Kapital" bezieht sich auf das in der Satzung aufgeführte Anfangskapital eines Joint Venture, das aus den vereinbarten Beiträgen der Partner für die Tätigkeit des Joint Venture besteht, ausgenommen die dem Joint Venture gewährten Kredite.

3. "Registriertes Kapital" bedeutet das beim SKKI registrierte gesetzliche Kapital.

Art. 21: 1. Ein Joint Venture wird in Vietnam auf der Grundlage eines Joint-Venture-Vertrages zwischen einem vietnamesischen und einem ausländischen Partner (im folgenden als Joint-Venture-Partner bezeichnet) mit dem Ziel begründet, Produktions- oder sonstige Geschäftstätigkeiten in den verschiedenen Sektoren der vietnamesischen Wirtschaft zu betreiben.

In besonderen Fällen kann ein Joint Venture auf der Grundlage eines zwischen der Regierung der SR Vietnam und der Regierung eines ausländischen Staates vereinbarten Abkommens errichtet werden.

2. Ein Joint Venture wird nach den Grundsätzen der selbständigen wirtschaftlichen Rechnungsführung in Úbereinstimmung mit dem Joint-Venture-Verrag, der Satzung des Joint Venture und den Gesetzen der SR Vietnam tätig.

Art. 22: Nach Erteilung der Investitionslizenz und der Urkunde über die Registrierung der Satzung durch das SKKI ist ein Joint Venture formell errichtet.

Art. 23: Der Antrag auf Investitionen ist von einem oder beiden der Joint-Venture-Partner zu unterzeichnen und beim SKKI zur Prüfung und Genehmigung einzureichen. Dem Antrag sind folgende Dokumente beizufügen:
(a) der Joint-Venture-Vertrag:
(b) die Machbarkeitsstudie;
(c) die Satzung des Joint Venture;
(d) sonstige vom SKKI zur Aufklärung bestimmter Fragen im Zusammenhang mit den oben genannten Dokumenten angeforderte Informationen; und
(e) der Antrag auf Gewährung von Vergünstigungen, soweit vorgesehen.

Art. 24: Ein Joint-Venture-Vertrag hat folgende Grundsatzklauseln zu enthalten:

(a) Daten bezüglich der Joint-Venture-Partner, wie Staatsangehörigkeit und Adressen und ihre bevollmächtigten Vertreter;

(b) Geschäftsnamen des Joint Venture, dessen voraussichtliche Adresse und eine Beschreibung der Geschäftstätigkeit;

(c) Das Gesamtinvestitionskapital, das gesetzliche Kapital, das registrierte Kapital, die Kapitaleinlagen jeder der Parteien, der Plan für die Kapitaleinlagen, die Bedingungen und das Verfahren für die Abtretung von Investitionskapital; 
(d) eine Liste der Quantität und Qualität der Grundausrüstung und der Materialien, die für die Geschäftstätigkeit notwendig sind, und deren Bezugsquelle; die Spezifikation der Quantität und Qualität der Produkte und deren Vermarktung; bei Dienstleistungsverträgen sind die Sätze für das Entgelt in konvertierbarer und in vietnamesischer Währung auszudrücken;

(e) die Laufzeit des Joint Venture und die Voraussetzungen seiner Beendigung und Auflösung;

(f) die Grundsätze der Finanzverwaltung, Buchführung und Rechnungsprüfung, einschließlich der Amortisierung des Anlagevermögens, den Satz der Zuteilung an die notwendigen Fonds und die Versicherung des Vermögens des Joint Venture;

(g) die Zahl der Mitglieder und die Zusammensetzung des Verwaltungsrates, die Zahl der von jedem Partner zu ernennenden Direktoren, die Befugnisse und Verantwortlichkeiten des Verwaltungsrates, des Vorsitzenden des Verwaltungsrates, des Generaldirektors und der stellvertretenden Generaldirektoren;

(h) den Satz für die Verteilung von Gewinn und Verlust zwischen den Partnern des Joint Venture;

(i) die Arbeitsverhältnisse im Joint Venture;

(j) die Trainingsprogramme für Manager, für das technische und Geschäftspersonal und die Arbeiter;

(k) das Verfahren zur Beilegung von Streitigkeiten zwischen den Joint-Venture-Partnern, die zwischen ihnen bei Ausführung des Joint-Venture-Vertrages entstehen, und das im Streitfalle anwendbare Recht;

(l) die Sprache des Joint-Venture-Vertrages; und

(m) die Voraussetzung für das Wirksamwerden des Joint-Venture-Vertrages.

Art. 25: Die Satzung eines Joint Venture hat folgende Grundsatzklauseln zu enthalten:

(a) den vollständigen Namen und die Adresse der Joint-Venture-Partner und ihrer Vertreter;

(b) den Geschäftsnamen des Joint Venture und dessen Sitz;

(c) die Beschreibung der beabsichtigten Produktions- und Geschäftstätigkeit des Joint Venture, die Ziele und den Gang der Produktion, des Betriebes und des Absatzes der Produkte;

(d) die beabsichtigte Zeitdauer des Joint Venture;

(e) das Gesamtinvestitionskapital, das gesetzliche Kapital und das Verfahren der Kapitaleinlage hinsichtlich des gesetzlichen Kapitals des Joint Venture;

(f) der Anteil der Beiträge jedes Joint-Venture-Partners zum gesetzlichen Kapital;

(g) Organisation und Zusammensetzung des Verwaltungsrates und die Organisation der Geschäftsführung des Join Venture;

(h) die Vertreter des Joint Venture vor Gerichten und Staatsbehörden;

(i) die Grundsätze für Finanzangelegenheiten, Buchführung und Rechnungsprüfung; 
(j) den Satz für die Verteilung von Gewinn und Verlust zwischen den Joint-VenturePartnem;

(k) die Voraussetzungen und das Verfahren für die Auflösung eines Joint Venture; und

(l) das Verfahren für die Abänderung der Satzung des Joint Venture.

Art. 26: 1. Das SKKI hat seine Entscheidung innerhalb dreier Monate vom Zeitpunkt des Eingangs des Antrages auf Erteilung einer Investitionslizenz an den Joint-Venture-Partnem mitzuteilen.

2. Wenn das SKKI von den Joint-Venture-Partnern zusätzliche Dokumente oder die Änderung bestirnmter Klauseln des Vertrages und/oder der Satzung verlangt, hat das in schriftlicher Form innerhalb eines Monats vom Zeitpunkt des Eingangs des Antrages an zu erfolgen.

Wenn von den Joint-Venture-Partnem eine schriftliche Antwort nicht innerhalb von fünfundvierzig Tagen nach Eingang einer solchen Aufforderung abgegeben wird, ist der Antrag auf Erteilung einer Investitionslizenz als gegenstandslos anzusehen. Bei einer unangemessenen Antwort der Joint-Venture-Partner ist die zur Erlangung einer befriedigenden Antwort zusätzlich erforderliche Zeit von der für die Prüfung und Bestätigung nach Absatz 1 dieses Artikels festgesetzten Zeitdauer auszunehmen.

3. Wenn dem Antrag stattgegeben wird, ist eine Investitionslizenz und eine Urkunde über die Registrierung der Satzung des Joint Venture den Joint-Venture-Partnem zu erteilen. Ausfertigungen dieser beiden Dokumente sind den Zentralbehörden und dem zuständigen Volkskomitee der Provinz, der Stadt oder der Sonderzone zu übersenden. Die Investitionslizenz und die Urkunde über die Registrierung der Satzung des Joint Venture ist im Amtsblatt der Regierung der SR Vietnam bekanntzugeben.

Art. 27: Vom Zeitpunkt der Erteilung der Investitionslizenz und der Urkunde über die Registrierung der Satzung des Joint Venture an tritt der Joint-Venture-Vertrag in Kraft und erlangt das Joint Venture den Status einer juristischen Person.

Art. 28: Veränderungen der Klauseln eines Joint-Venture-Vertrages und der Satzung des Joint Venture, welche die Joint-Venture-Partner vereinbaren, werden erst nach Genehmigung durch das SKKI wirksam.

Art. 29: Die Joint-Venture-Partner haben ihre jeweiligen Einlagen zum gesetzlichen Kapital des Joint Venture in Ubereinstimmung mit den Vorschriften des Artikel 7 und 8 des Investitionsgesetzes vorzunehmen. 
Die Bewertung der Kapitaleinlagen eines jeden Partners richtet sich nach der Vereinbarung beider Partner unter Berücksichtigung der Preise des internationalen Marktes zur Zeit der Vornahme der Einlagen.

Das SKKI ist befugt, diese Bewertung zu überprüfen und von den Partnem zu verlangen, die Kapitaleinlagen unter Beachtung der Bestimmungen dieses Artikels neuzubewerten.

Art. 30: Die von den Joint-Venture-Partnem vorzunehmenden Einlagen zum gesetzlichen Kapital können als einmalige Leistung zur Zeit der Errichtung des Joint Venture oder in Raten innerhalb einer von beiden Partnem vereinbarten angemessenen Frist erfolgen.

Die Art der Kapitaleinalge zum gesetzlichen Kapital ist ausdrücklich in der Satzung des Joint Venture anzugeben.

Art. 31: Im Verlauf seiner Tätigkeit ist es einem Joint Venture untersagt, das gesetzliche Kapital herabzusetzen. Jede Erhöhung des gesetzlichen Kapitals setzt eine Vereinbarung zwischen den Joint-Venture-Partnem voraus und bedarf der Registrierung beim SKKI.

Art. 32: Jeder der Partner hat das Recht, seine Kapitaleinalge in das Joint Venture abzutreten; er hat dabei dem anderen Partner des Joint Venture ein Erstangebot zu machen. Kommt eine Einigung zwischen den Partnem über die Abtretungsbedingungen nicht zustande, hat der abtretungswillige Partner das Recht, sein Investitionskapital einer dritten Partei zu übertragen, vorausgesetzt, die UUbertragungsbedingungen sind nicht günstiger als jene, die dem anderen Partner angeboten wurden. Die Übertragung wird erst nach einstimmigem schriftlichen Beschluß des Verwaltungsrates des Joint Venture und nach Genehmigung durch das SKKI wirksam.

Art. 33: Ein Joint Venture ist als Gesellschaft mit beschränkter Haftung zu errichten; die Haftung jedes Partners gegenüber dem anderen Partner, gegenüber dem Joint Venture und gegenüber dritten Parteien ist auf das gesetzliche Kapital beschränkt.

Art. 34: Das höchste Gremium eines Joint Venture ist der Verwaltungsrat. Der Verwaltungsrat hat die Befugnis, Beschlüsse über alle wesentlichen Angelegenheiten des Joint Venture zu fassen.

Art. 35: 1. Die Zahl der Mitglieder des Verwaltungsrates, die Sitzverteilung im Verwaltungsrat, die Emennung der Mitglieder und die Bestimmung des Vorsitzenden des Verwaltungsrates, des Generaldirektors und der stellvertretenden Generaldirektoren richtet sich nach Artikel 12 des Investitionsgesetzes. Der Vorsitzende des Verwaltungsrates kann gleichzeitig der Generaldirektor des Joint Venture sein. 
2. Die Amtszeit der Mitglieder des Verwaltungsrates ist von den Joint-Venture-Partnern durch Vereinbarung zu bestimmen, darf jedoch fünf Jahre nicht überschreiten.

Art. 36: Die Zeitabstände für die Einberufung von Sitzungen des Verwaltungsrates sind von den Partnern in der Satzung des Joint Venture zu bestimmen; der Verwaltungsrat muß jedoch mindestens einmal jährlich zusammentreten. Die Sitzungen des Verwaltungsrates werden vom Vorsitzenden des Verwaltungsrates einberufen.

Art. 37: Jede Sitzung des Verwaltungsrates erfordert die Anwesenheit von mehr als zwei Dritteln seiner Mitglieder.

Mitglieder des Verwaltungsrates können durch schriftliche Vollmacht Vertreter zur Teilnahme an den Sitzungen des Verwaltungsrates entsenden und ihnen ihre Stimme übertragen.

Art. 38: Beschlüsse über die nachfolgend aufgeführten Angelegenheiten unterliegen dem Grundsatz der Einstimmigkeit:

1. Produktions- und Geschäftspläne des Joint Venture, sein Betriebsbudget und Kreditaufnahmen;

2. Änderungen und Ergänzungen der Satzung des Joint Venture und wesentliche Änderungen in der Organisation und Tätigkeit des Joint Venture einschließlich der Veränderung des registrierten Geschäftsziels und Geschäftsablaufs, die Erhöhung des gesetzlichen Kapitals, die Kapitalabtretung, Verlängerung der Laufzeit oder vorübergehende Einstellung der Tätigkeit, Fusion und Auflösung;

3. Ernennung, Ersetzung und Entlassung des Vorsitzenden des Verwaltungsrates, des Generaldirektors und der stellvertretenden Generaldirektoren oder anderer Führungskräfte des Joint Venture.

Beschlüsse des Verwaltungsrates über sonstige Angelegenheiten sind wirksam, wenn sie mit einer Mehrheit von zwei Dritteln der anwesenden Mitglieder des Verwaltungsrates gefaßt worden sind.

Art. 39: Der Generaldirektor und die stellvertretenden Generaldirektoren sind für die Führung und Leitung der laufenden Geschäfte des Joint Venture verantwortlich. Hat ein Joint Venture mehr als einen stellvertretenden Generaldirektor, so hat der Verwaltungsrat einen davon zum ersten stellvertretenden Generaldirektor zu bestellen. Ist der Generaldirektor ein Ausländer, muß der erste stellvertretende Generaldirektor ein vietnamesischer Bürger sein. 
Der Verwaltungsrat bestimmt die jeweiligen Verantwortlichkeiten und Befugnisse des Generaldirektors und des ersten stellvertretenden Generaldirektors. Der Generaldirektor und der erste stellvertretende Generaldirektor sind dem Verwaltungsrat gegenüber für die Tätigkeit des Joint Venture verantwortlich.

Art. 40: Der in diesem Kapitel erwähnte Technologietransfer bedeutet die Einbringung von erforderlicher Technologie in ein Joint Venture durch einen der Partner in der Form einer Kapitaleinlage oder den Verkauf der Technologie an das Joint Venture durch eine dritte Partei.

Art. 41: 1. Die in ein Joint Venture eingebrachte Technologie muß für das Joint Venture notwendig und geeignet sein, wesentliche sozio-ökonomische Wirkungen zu äußern oder die Wettbewerbsfähigkeit seiner Produkte auf internationalen Märkten zu steigern.

2. die transferierte Technologie hat folgende Voraussetzungen zu erfüllen:

(a) sie muß geeignet sein, eine beträchtliche Verbesserung des Aussehens und der Qualität der Produkte und die Steigerung des Absatzes zu bewirken;

(b) sie muß geeignet sein, neue, in Vietnam dringend benötigte Produkte oder Importersatzwaren zu erzeugen;

(c) ihre Anwendung bewirkt merkliche Einsparungen an Rohstoffen und Energie.

Art. 42: Die Regierung der SR Vietnam schützt das gewerbliche Eigentum an der transferierten Technologie. Die in dem Vertrag über den Technologietransfer vereinbarten Rechte und Pflichten der Parteien müssen mit den in dieser Hinsicht in Vietnam geltenden Vorschriften und der internationalen Praxis in Einklang stehen.

Jeder Vertrag über den Technologietransfer in bezug auf ein Joint Venture in der Form einer Kapitaleinlage ist dem Antrag auf Erteilung einer Investitionslizenz beizufügen.

Art. 43: Die Laufzeit des Joint Venture ist zwischen den Joint-Venture-Partnern im JointVenture-Vertrag zu vereinbaren. Grundsätzlich soll die Laufzeit eines Joint Venture nicht zwanzig Jahre überschreiten. Im Hinblick jedoch auf Projekte über die Ausbeutung natürlicher Ressourcen und auf solche, die umfangreiche Investitionen und lange Zeiträume für die Anlegung oder Rückgewinnung des investierten Kapitals erfordern, können die JointVenture-Partner eine längere Laufzeit vereinbaren, die jedoch fünzig Jahre nicht überschreiten darf.

Art. 44: Die Laufzeit eines Joint Venture beginnt im Zeitpunkt der Erteilung der Investitionslizenz und der Urkunde über die Registrierung der Satzung des Joint Venture. 
Art. 45: Wenn die Joint-Venture-Partner übereinkommen, die Laufzeit eines Joint Venture über den in der Investitionslizenz genannten Zeitraum hinaus auszudehnen, haben sie einen entsprechenden Antrag spätestens sechs Monate vor Beendigung der Laufzeit des Joint Venture beim SKKI einzureichen.

Innerhalb von dreißig Tagen vom Zeitpunkt des Eingangs des Antrages an hat das SKKI die Joint-Venture-Partner über seine Entscheidung zu unterrichten. Wenn dem Anrag stattgegeben wird, können die Joint-Venture-Partner ihre Tätigkeit ohne erneute Registrierung fortsetzen.

Art. 46: Ein Joint Venture kann vor Beendigung der im Joint-Venture-Vertrag festgelegten Laufzeit seine Tätigkeit in folgenden Fällen einstellen und aufgelöst werden:

1. Vorliegen einer im Vertrag bezeichneten höheren Gewalt, welche die Unmöglichkeit der Erfüllung zur Folge hat;

2. Nichterfüllung der vertraglichen Pflichten eines der Partner mit der Folge, daß das Joint Venture seine Tätigkeit nicht fortsetzen kann;

3. Verluste des Joint Venture von einem Ausmaß, daß es zur Fortsetzung seiner Tätigkeit nicht mehr in der Lage ist;

4. durch die Tätigkeit des Joint Venture verursachte schwerwiegende Umweltverschmutzung, ohne daß eine Lösung für ihre rechtzeitige Kontrolle absehbar ist;

5. Übertragung des gesamten Kapitalanteils am Joint Venture durch einen der JointVenture-Partner auf den anderen Partner;

6. sonstige im Joint-Venture-Vertrag vorgesehene Fälle der Auflösung.

Art. 47: Die Auflösung eines Joint Venture vor Ablauf seiner Tätigkeitsdauer ist durch den Verwaltungsrat zu beschließen und dem SKKI zur Genehmigung mitzuteilen.

Das SKKI hat die Befugnis, ein Joint Venture vor Ablauf seiner Tätigkeitszeit aufzulösen, wenn die Tätigkeit des Joint Venture in dem in der Satzung und in der Investitionslizenz angegebenen Ziel und Rahmen seiner Tätigkeit abweicht.

Art. 48: Der Verwaltungsrat hat spätestens innerhalb von sechs Monaten vor Beendigung der Laufzeit des Joint Venture oder nach dem Beschluß über die vorzeitige Auflösung des Joint Venture einen aus mindestens drei Mitgliedern bestehenden Liquidationsausschuß einzusetzen und die von dem Ausschuß zu erfüllenden Aufgaben zu bestimmen. Die Mit- 
glieder des Liquidationsausschusses können aus der Mitte des Personals des Joint Venture oder betriebsfremder Experten gewählt werden.

Alle durch das Liquidationsverfahren entstehenden Kosten sind vom Joint Venture zu tragen; ihre Begleichung geht allen anderen Verbindlichkeiten des Joint Venture vor.

Art. 49: Der Liquidationsausschuß hat das SKKI über den Zeitpunkt zu unterrichten, in dem er tatsächlich zusammentritt und seine Arbeit aufnimmt. Von diesem Zeitpunkt an vertritt der Liquidationsausschuß das Joint Venture bei allen Handlungen im Zusammenhang mit Rechtsverfahren vor Gericht und/oder vor Verwaltungsbehörden.

Die Amtszeit des Liquidationsausschusses soll sechs Monate nicht überschreiten. Soweit erforderlich kann diese Frist verlängert werden, jedoch nicht über ein Jahr hinaus.

Art. 50: Das Vermögen des Joint Venture ist nach dem aktuellen Zustand im Zeitpunkt der Liquidation zu bewerten. Wenn der Wert dieses Vermögens das gesetzliche Kapital übersteigt, ist der Zwischenbetrag als Gewinn des Joint Venture zu betrachten und der Gewinnbesteuerung zugrundezulegen.

Art. 51: Nach Abschluß seiner Tätigkeit hat der Liquidationsausschuß einen Liquidationsbericht dem Verwaltungsrat und danach dem SKKI spätestens innerhalb von zwei Monaten vorzulegen.

Nach Ablauf der in Artikel 49 vorgesehenen Jahresfrist hat das SKKI die Tätigkeit des Liquidationsausschusses einzustellen, selbst wenn zwischen den Joint-Venture-Partnem weiterhin Streit über das Liquidationsverfahren besteht. Diese Streitigkeiten sind in Übereinstimmung mit den Vorschriften des Artikels 53 dieses Dekrets beizulegen.

Das SKKI hat daraufhin eine Entscheidung über die Löschung der Investitionslizenz zu treffen und diese Entscheidung den zuständigen Behörden mitzuteilen. Danach hat der Liquidationsausschuß das Original der Investitionslizenz dem SKKI zurückzureichen.

Art. 52: Nach Auflösung des Joint Venture sind die Kontenbücher und anderen erhebliche Dokumente beim SKKI zu hinterlegen.

Art. 53: Streitigkeiten zwischen den Joint-Venture-Partnern im Verlauf der Durchführung des Joint-Venture-Vertrages sind im Verhandlungswege und durch gütliches Einvernehmen zwischen den Partnem beizulegen.

Läßt sich ein Einvernehmen nicht erzielen, können die streitenden Parteien den Streit einem der folgenden Organe unterbreiten: 
dem Außenhandelsarbitragekomitee bei der Kammer für Handel und Industrie der SR Vietnam oder der Arbitragebehörde eines dritten Staates oder einer intemationalen Arbitragebehörde oder einem Arbitragekomitee, auf dessen Errichtung sich die Parteien geeinigt haben.

Die Joint-Venture-Partner haben die Vereinbarung über die Form der Arbitrage und das Arbitrageorgan in den Joint-Venture-Vertrag aufzunehmen.

\section{Kapitel IV: Privatunternehmen mit 100 \%iger ausländischer Kapitaleinlage}

Art. 54: Ein Untemehmen mit $100 \%$ igem ausländischen Kapital steht vollständig im Eigentum einer ausländischen Organisation oder Privatperson und wird von der genannten Organisation oder Privatperson allein errichtet und geleitet.

Art. 55: Ein Untemehmen mit $100 \%$ igem ausländischen Kapital ist als Gesellschaft mit beschränkter Haftung des vietnamesischen Rechts nach dem Recht der SR Vietnam zu errichten.

Art. 56: Die Laufzeit des Unternehmens mit 100 \%igem ausländischen Kapital entspricht der für Joint Ventures in Artikel 43 dieses Dekrets bestimmten Laufzeit.

Art. 57: Bei Stellung eines Antrages auf Erteilung einer Investitionslizenz hat der Antragsteller dem SKKI folgende Dokumente vorzulegen:

(a) eine Machbarkeitsstudie als Grundlage der Entscheidung des Antragstellers für die beabsichtigte Investition, worin ausdrücklich die wirtschaftliche und finanzielle Dienlichkeit des Projekts begründet wird;

(b) die Satzung der Organisation oder der Nachweis für die Geschäftsfähigkeit der Privatperson, welche die Investition beabsichtigt, zusammen mit den notwendigen Informationen über den Investor, insbesondere über seine finanziellen Verhältnisse;

(c) Garantien für die Eignung des Investors, langfristig Geschäfte in Vietnam zu tätigen;

(d) die Satzung des in Vietnam zu errichtenden Unternehmens;

(e) Antrag auf Vergünstigungen, soweit vorgesehen; und

(f) sonstige vom SKKI angeforderte Dokumente.

Art. 58: Die Satzung des Unternehmens mit $100 \%$ igem ausländischen Kapital hat folgende Grundsatzklauseln zu enthalten:

(1) Geschäftsnamen und Sitz des Unternehmens;

(2) Beschreibung der Produktions- und Geschäftstätigkeit des Unternehmens;

(3) Gesamtbetrag der Kapitalinvestition und das gesetzliche Kapital des Unternehmens; 
(4) die beabsichtigte Laufzeit des Unternehmens;

(5) die Organisation der Führung der laufenden Geschäfte und die Leitung der Geschäftstätigkeit; den Vertreter des Unternehmens vor vietnamesischen Gerichten und Behörden;

(6) die Grundsätze für Finanzangelegenheiten, die Buchführung und Rechnungsprüfung; und

(7) die Voraussetzungen und das Verfahren für die Auflösung des Unternehmens.

Art. 59: Die Prüfung eines Antrages wegen Investitionen und die Erteilung einer Investitionslizenz an ein Unternehmen mit $100 \%$ igem ausländischen Kapital erfolgt nach Kapitel III dieses Dekrets in derselben Weise wie bei einem Joint Venture.

Art. 60: Änderungen der Satzung eines Unternehmens mit $100 \%$ igem ausländischen Kapital werden erst nach Genehmigung durch das SKKI wirksam.

Art. 61: Der im Ausland wohnhafte Inhaber eines Unternehmens hat einen ordnungsgemä $ß$ bevollmächtigten Vertreter in Viemam zu bestellen und die in der Investitionslizenz aufgeführten Bestimmungen einzuhalten.

Art. 62: Das SKKI hat die Befugnis, eine Entscheidung über die vorübergehende Einstellung der Tätigkeit des Unternehmens mit $100 \%$ igem ausländischen Kapital oder dessen Auflösung vor Ablauf der Geschäftsfrist zu treffen, wenn die Tätigkeit des Untemehmens von den in der Satzung des Unternehmens und in der Investitionslizenz aufgeführten Zielen abweicht oder dadurch eine schwerwiegende Umweltverschmutzung verursacht wird, ohne $\mathrm{da} ß$ eine Lösung für ihre rechtzeitige Kontrolle absehbar ist.

\section{Kapitel V: Geschäftsorganisation}

Art. 63: Die Parteien eines Unternehmens-Kooperations-Vertrages und Unternehmen mit ausländischem Investitionskapital haben das uneingeschränkte Recht, über ihre eigenen Produktions- und Geschäftsprogramme und -pläne zu entscheiden. Die viemamesischen Staatsbehörden erlegen diesen Parteien keine Planziele auf.

Art. 64: In der Machbarkeitsstudie, welche dem Antrag auf Erteilung einer Investitionslizenz beizufügen ist, haben die Parteien eines Unternehmens-Kooperations-Vertrages und Unternehmen mit ausländischem Investitionskapital ausdrücklich folgendes anzuführen:

im Hinblick auf die Kapitalstruktur: die Abfolge der Aufbaustufen und die Quelle der Bedarfsdeckung für Ausrüstung, Maschinen, Baumaterial, Wasser, Elektrizität und Brennstoff usw.; 
im Hinblick auf den Produktionsplan: die Quelle der Bedarfsdeckung für Rohstoffe und andere Materialien und Brennstoffe zu Produktionszwecken;

im Hinblick auf den Absatz der Produkte: Export und Absatz der Produkte auf dem Binnenmarkt, soweit beabsichtigt.

Art. 65: Für Ausrüstung, Maschinen, Transportfahrzeuge oder Rohstoffe und andere Materialien, die von einem ausländischen Partner als Teil seines Kapitalbeitrages nach Vietnam zu importieren sind, bedarf es nur eines Antrages auf Import.

Importe von Ausrüstung, Maschinen, Ersatzteilen, Transportfahrzeugen, Rohstoffen und anderen Materialien, Brennstoffen usw. zur Deckung des Produktionsbedarfs eines vom SKKI genehmigten Unternehmens-Kooperations-Vertrages unterliegen entweder einer einmaligen Gesamtimportquote oder einer jährlichen Importquote, je nachdem, was die Parteien des Vertrages vorziehen. Soweit erforderlich, können Zusätze oder Berichtigungen einer Importquote unter Berücksichtigung eines vom Unternehmen unterbreiteten entsprechenden vernünftigen Vorschlages erfolgen.

Importlizenzen werden in Übereinstimmung mit den geltenden Regelungen über Exportund Importkontrollen erteilt.

Bei gleichen Handelsbedingungen genießen in Vietnam verfügbare Produkte als Importersatz den Vorzug; in diesen Fällen sind mit den betreffenden vietnamesischen Wirtschaftsorganisationen entsprechend dem vietnamesischen Recht Lieferverträge zu vereinbaren; die Zahlung erfolgt entweder in ausländischer oder in vietnamesischer Währung, je nachdem, um welches Produkt es sich handelt.

Art. 66: Die Parteien eines Unternehmens-Kooperations-Vertrages und Unternehmen mit ausländischem Investitionskapital haben das Recht, die Produkte in Ubereinstimmung mit den jährlichen Exportplänen entweder selbst zu exportieren oder diese Exporte Agenturen eines ausländischen Partners oder einer vietnamesischen Außenhandelsgesellschaft zu übertragen, je nachdem, was die Parteien vereinbart haben.

Art. 67: Im Hinblick auf Produkte, die auf dem vietnamesischen Markt absetzbar sind, können die Parteien eines Unternehmens-Kooperations-Vertrages und Unternehmen mit. ausländischem Investitionskapital die Verkäufe entweder selbst vornehmen oder sie den betreffenden vietnamesischen Handelsorganisationen durch den Abschluß von Wirtschaftsverträgen gemäß vietnamesischem Recht überlassen. 


\section{Kapitel VI: Arbeitsbeziehungen in Unternehmen mit ausländischem Investi- tionskapital}

Art. 68: Arbeitsbeziehungen in Unternehmen mit ausländischem Investitionskapital einschließlich Einstellung, Arbeitszeit, Arbeitsschutz, Arbeitsdisziplin, Löhne und Gehälter, Gratifikationen, Sozialversicherungsleistungen und die Beilegung von Arbeitsstreitigkeiten sind durch Arbeitsverträge zu regeln.

Art. 69: Ein Unternehmen mit ausländischem Investitionskapital kann Arbeitsverträge folgender Art abschließen:

(a) freie Auswahl der Arbeiter und Abschluß gesonderter Verträge mit den einzelnen Arbeitern, die durch das vietnamesische Arbeitsverwaltungsbüro vermittelt worden sind;

(b) Abschluß eines kollektiven Arbeitsvertrages mit der Gewerkschaft des Unternehmens oder mit einer Gesellschaft für Arbeitsvermittlung oder Investitionsdienste;

(c) Abschluß eines kollektiven Arbeitsvertrages gemäß Buchstaben (b) mit nachträglichem Abschluß gesonderter Arbeitsverträge mit den einzelnen Arbeitern.

Unabhängig von der Art der Verträge, die mit den Arbeitern abgeschlossen werden, muß das betreffende Unternehmen mit ausländischem Investitionskapital die geltende Arbeitsgesetzgebung Vietnams beachten.

Art. 70: Die Löhne der Arbeiter sind von den Parteien unter Berücksichtigung der jeweiligen Qualifikationen, Arbeitsgebiete, Arbeitsleistungen und Arbeitsqualitäten zu vereinbaren. Die Löhne sollen einen angemessenen Lebensstandard ähnlich dem aufgrund von Arbeitseinkünften in ausländischen Staaten unter vergleichbaren Arbeitsbedingungen erzielten Niveau gewährleisten.

Art. 71: Das Unternehmen mit ausländischem Investitionskapital hat die Löhne und Gehälter entweder direkt an die Beschäftigten oder an die Organisation, welche den kollektiven Arbeitsvertrag mit dem Unternehmen geschlossen hat, zu zahlen, je nach den in den Verträgen vereinbarten Bedingungen.

Art. 72: Arbeiter eines Unternehmens mit ausländischem Investitionskapital haben das Recht, eigene Gewerkschaften zur Sicherung der Arbeitsbeziehungen und Vertretung der Arbeiter in allen wesentlichen Fragen gegenüber dem Unternehmen zu gründen.

Das Unternehmen hat die Voraussetzungen zu schaffen, die zur Wahmehmung der Aufgaben von Gewerkschaften erforderlich sind. 


\section{Kapitel VII: Finanzangelegenheiten}

Art. 73: 1. Ein Unternehmen mit ausländischem Investitionskapital und der ausländische Partner einer vertraglichen Unternehmens-Kooperation haben Körperschaftsteuem auf den Gewinn nach einer der folgenden Kategorien abzuführen:

(a) Vorzugskategorie: $15 \%$ bis $20 \%$ des tatsächlichen Gewinns; und

(b) Standardkategorie: $21 \%$ bis $25 \%$ des tatsächlichen Gewinns.

2. Die Vorzugskategorie bezieht sich auf Investitionsprojekte der in Artikel 3 des Investitionsgesetzes aufgeführten Art, welche zwei der im folgenden aufgeführten Merkmale erfüllen:

(a) das gesetzliche Kapital beträgt nicht weniger als zehn Millionen US-Dollar;

(b) die transferierte Technologie erfüllt mindestens zwei der in Absatz 2 des Artikels 41 dieses Dekrets aufgeführten Erfordemisse;

(c) das Unternehmen exportiert mindestens $80 \%$ seiner Produkte und erzielt mindestens $80 \%$ seiner Gewinne aus Dienstleistungen in ausländischer Währung;

(d) die Profitrate des Unternehmens hält sich im unteren Rahmen im Vergleich zu den durchschnittlich in anderen Projekten derselben Branche erzielten Gewinnen;

(e) das Investitionsprojekt ist in einer Region mit ungünstigen natürlichen und sozialen Bedingungen angesiedelt; und

(f) die Investitionen werden innerhalb der ersten fünf Jahre nach Inkrafttreten des Investitionsgesetzes vorgenommen.

3. Das SKKI trifft nach den in Absatz 2 und 3 dieses Artikels aufgestellten Merkmalen gegenüber jedem einzelnen Projekt eine Entscheidung für einen bestimmten Steuersatz innerhalb des in Absatz 1 dieses Artikels festgelegten Steuerrahmens.

4. Bei Projekten über die Ausbeutung von Öl und Gas und sonstiger seltener und hochwertiger natürlicher Ressourcen hat das SKKI von Fall zu Fall eine Entscheidung für Körperschaftsteuern von mehr als $25 \%$ des tatsächlichen Gewinns unter Berücksichtigung der intemationalen Praxis und der Vorstellung des ausländiśschen Investors zu treffen.

Art. 74: Bei einem Joint Venture der Vorzugskategorie kann das SKKI unter Beachtung der in Absatz 2 des Artikels 73 dieses Dekrets aufgeführten Merkmale Steuerbefreiung für einen maximalen Zeitraum von zwei Jahren von dem Zeitpunkt an, in dem das Joint Venture Gewinne erzielt, gewähren und anschließend für einen maximalen Zeitraum von zwei Jahren Steuernachlaß von 50 \% der Körperschaftsteuer.

Art. 75: 1. In Ausnahmefällen kann das SKKI zum Anreiz für Investitionen Joint Ventures folgende finanzielle Vergünstigungen gewähren:

(a) Körperschaftsteuem von $10 \%$ bis zu $14 \%$ des tatsächlichen Gewinns; 
(b) Befreiung von der Körperschaftsteuer für einen Zeitraum von höchstens vier Jahren und anschließend einen 50 \%igen Steuernachlaß für einen Höchstzeitraum von vier Jahren nach dem ersten gewinnträchtigen Jahr.

2. Um Ansprüche auf besondere finanzielle Vergünstigungen geltend machen zu können, muß ein Joint Venture neben seiner in Artikel 73 dieses Dekrets genannten Vorzugskategorie solche Qualifikationen aufweisen, wie Tätigwerden unter außerordentlich schwierigen Bedingungen oder in abgelegenen Gebieten von sehr niedrigem Entwicklungsniveau oder Aktivitäten von außerordentlicher Bedeutung für die nationale Wirtschaft Vietnams trotz niedriger Profitrate.

Art. 76: Eine ausländische Wirtschaftsorganisation oder Privatperson hat eine Steuer auf die Rückführung des Gewinns ins Ausland zu den folgenden Sätzen zu entrichten:

1. eine ausländische Wirtschaftsorganisation oder Privatperson mit einem Kapitalbeitrag von über $50 \%$ des gesetzlichen Kapitals oder einem Investitionskapital von über 10 Millionen US-Dollar: $5 \%$ des Betrages, der für die Gewinnrückführung verwendet wird;

2. sonstige Organisationen oder Privatpersonen, die nicht unter Absatz 1 dieses Artikels fallen: $10 \%$ des Betrages, der für die Gewinnrückführung verwendet wird.

Art. 77: Jede ausländische Wirtschaftsorganisation und Privatperson, die einen Teil ihres Gewinnanteils für einen Zeitraum von drei Jahren oder darüber hinaus reinvestiert, hat einen Anspruch gegen die Steuerbehörde auf Rückzahlung der bereits auf den reinvestierten Gewinnanteil entrichteten Einkommensteuer.

Art. 78: Das für ein Unternehmen mit ausländischem Investitionskapital und Partnern einer vertraglichen Unternehmens-Kooperation geltende Steuerjahr deckt sich mit dem Kalenderjahr.

Das Unternehmen und die Partner einer vertraglichen Unternehmens-Kooperation können jedoch beim Finanzministerium der SR Vietnam beantragen, daß für Steuerzwecke ihnen gegenüber das auf die Buchführung anwendbare zwölfmonatige Finanzjahr gilt.

Art. 79: Das versteuerbare körperschaftliche Einkommen eines Unternehmens mit ausländischem Investitionskapital ist das Nettoeinkommen eines jeden Steuer jahres nach Abzug aller während dieses Jahres anfallenden Kosten und Ausgaben. Dieses Einkommen umfaßt das versteuerbare Einkommen des Haupt- und Zweigunternehmens, soweit vorhanden. 
Einnahmen und Ausgaben eines Unternehmens mit ausländischem Investitionskapital umfassen folgende Positionen:

(a) Einnahmen aus Verkäufen von Produkten oder aus Dienstleistungen und alles übrige aus der Geschäftstätigkeit erzielte Einkommen;

(b) betreffend Ausgaben:

Kosten für Rohstoffe, sonstiges Material und Energie zur Herstellung von Haupt- und Nebenprodukten oder für Dienstleistungen; Löhne, Gehälter und Zusatzleistungen für ausländisches und vietnamesisches Personal und Arbeiter des Unternehmens;

Abschreibung des Anlagevermögens für Produktion und Geschäftstätigkeit; Satz und Höhe der Abschreibung richten sich nach der vom Ministerium für Finanzen der SR Vietnam zu genehmigenden Vereinbarung der Partner;

Erwerb oder Nutzung technischer Dokumente aufgrund von Lizenzen, Patenten, Industrielizenzen und Kosten für technische Dienstleistungen;

Betriebskosten;

Steuern und steuergleiche Verbindlichkeiten;

Kosten und Ausgaben, die direkt mit dem Absatz von Produkten und der Erbringung von Dienstleistungen verbunden sind;

Beiträge zum Sozialversicherungsfonds;

Kosten für die Versicherung des Vermögens des Unternehmens;

Verlustübertragungen aus dem Vorjahr; und

sonstige Ausgaben, die insgesamt $5 \%$ der Gesamtsumme der Ausgaben nicht überschreiten.

Art. 80: Bei Unternehmens-Kooperationen wird die Methode der Bewertung von Gewinnen vom SKKI unter Berücksichtigung der Natur der Kooperation und der Vorstellungen der betreffenden Partner bestimmt.

Bei Arbeitsteilungen in der Produktion werden Einkommensteuern und Entgelte des vietnamesischen Partners für Nutzungsrechte an Land, Binnengewässern und dem Meer und sonstige Gebühren dessen Anteil an der Produktion hinzugerechnet.

Art. 81: Ein Unternehmen mit ausländischem Investitionskapital und die Partner einer vertraglichen Unternehmens-Kooperation haben das Recht auf Befreiung von Importzöllen in folgenden Fällen:

(a) Import von Ausrüstung, Maschinen, Ersatzteilen und Geschäftsgeräten einschließlich Transportfahrzeugen und anderen Materialien als Kapitaleinlage zum Unternehmen oder zur Unternehmens-Kooperation;

(b) Import von Ausrüstung, Maschinen, Ersatzteilen und sonstigen Materialien zur Einbringung in den Fonds des Unternehmens als Bestandteil des Gesamtinvestitionskapitals; und 
(c) Import von Rohstoffen, Ersatzteilen, Hilfsmitteln und sonstigen Materialien zur Produktion von Exporterzeugnissen.

Alle in den obigen Buchstaben (a), (b) und (c) aufgeführten importierten Produkte unterliegen, wenn sie in Vietnam veräußert oder verkauft werden, nach den Bestimmungen der in Vietnam geltenden Gesetze dem Importzoll und der Mehrwertsteuer.

Art. 82: Unternehmen mit ausländischem Investitionskapital oder Partner einer vertraglichen Unternehmens-Kooperation, die in Vietnam Dienstleistungen gegen Entgelt erbringen oder auf dem vietnamesischen Markt Produkte verkaufen, haben nach den Bestimmungen der vietnamesischen Gesetze Geschäfts- und Mehrwertsteuern zu entrichten.

Art. 83: Ausländer und Vietnamesen, die in einem Unternehmen mit ausländischem Investitionskapital beschäftigt oder im Rahmen einer vertraglichen Unternehmens-Kooperation tätig sind, haben persönlich Einkommensteuem nach den Vorschriften der vietnamesischen Gesetze zu entrichten.

Art. 84: Land, Forsten, das Meer und andere Naturreichtümer innerhalb Vietnams unterfallen der Souveränität der SR Vietnam.

Wenn Gebühren für die Nutzung von Land, Binnengewässem oder das Meer nicht, wie nach Artikel 7 des Investitionsgesetzes vorgesehen, Teil der Kapitaleinlage des vietnamesischen Parmers ist, haben Untemehmen mit ausländischem Investitionskapital oder die Partner vertraglicher Unternehmens-Kooperationen die anfallenden Gebühren zu zahlen. Die Gebühren werden in Übereinstimmung mit der internationalen Praxis und dem Grundsatz des beiderseitigen Vorteils festgesetzt.

Pachtzinsen für Land, Binnengewässer und das Meer, die von den Partnem einer vertraglichen Unternehmens-Kooperation zu entrichten sind, werden unter Berücksichtigung der in dem geographischen Gebiet vorherrschenden besonderen Verhältnisse bestimmt.

\section{Kapitel VIII: Devisenkontrolle}

Art. 85: Alle Kapitalfonds und Einkünfte eines Unternehmens mit ausländischem Investitionskapital in ausländischer oder vietnamesischer Währung sind auf ein Konto bei der Bank für Außenhandel in Vietnam oder Zweigniederlassung einer gemischt ausländischvietnamesischen Bank oder einer ausländischen Bank in Vietnam nach Genehmigung der Staatsbank von Vietnam einzuzahlen. 
Alle Einnahmen und Ausgaben des Untemehmens sind über diese Konten abzuwickeln. Soweit erforderlich, kann der ausländische Partner einer vertraglichen UnternehmensKooperation ein solches im vorhergehenden Absatz genanntes Konto eröffnen.

Art. 86: Ein Unternehmen mit ausländischem Investitionskapital hat das Recht, soweit erforderlich, Devisenguthaben in vietnamesische Währung zu konvertieren.

Art. 87: Abgesehen von den Sonderfällen wie der Produktion von Importersatzgütem oder Projekten im Bereich der Infrastruktur, müssen die Deviseneinnahmen des Unternehmens aus Exporten und anderen Quellen des Untemehmens entsprechend dem Grundsatz des Ausgleichs von Einnahmen und Ausgaben in ausländischer Währung ausreichen, um alle in ausländischer Währung zu leistenden Zahlungen vomehmen zu können, einschließlich der Rückfühnung von Gewinnen ins Ausland und der Zahlung von Löhnen, Gehältem und zusätzlichen Leistungen an die in dem Untemehmen beschäftigten vietnamesischen Angestellten und Arbeiter, auch wenn diese Zahlungen in vietnamesischer Währung erfolgen können.

Art. 88: 1. Ausländische Wirtschaftsorganisationen oder Einzelpersonen, die in Vietnam investieren, haben das Recht, ins Ausland zurückzuführen:

(a) den aus der Geschäftstätigkeit erzielten Gewinnanteil;

(b) alle ihnen für Dienstleistungen oder Technologietransfer zustehenden und genehmigten Zahlbeträge;

(c) Kapital und Zinsen der den Unternehmen gewährten Kredite; und

(d) Geldbeträge und Vermögenswerte, deren rechtmäßige Eigentümer sie sind.

Die obige Rückführung kann erst nach vollständiger Begleichung aller fälligen Steuerverbindlichkeiten erfolgen.

2. Bei Beendigung oder Auflösung des Unternehmens haben ausländische Wirtschaftsorganisationen und Einzelpersonen das Recht, ihre Kapitaleinlagen und jedes sonstige in das Unternehmen reinvestierte Kapital nach Begleichung aller Verbindlichkeiten zu repatriieren oder ins Ausland zu transferieren.

3. Die in diesem Artikel genannte Repatriierung und Rückführung von Kapital ins Ausland erfolgt grundsätzlich in drei gleichen Jahresraten. In Ausnahmefällen kann die Staatsbank von Vietnam jedoch gestatten, daß die Repatriierung oder der Transfer in Ratenzahlungen innerhalb eines kürzeren Zeitraumes erfolgt.

4. Wenn aus irgendwelchen Gründen der Betrag, dessen Repatriierung oder Transfer ins Ausland gemä $ß$ dem vorstehenden Absatz 2 erfolgen soll, höher ist als derjenige der von einer ausländischen Wirtschaftsorganisation oder Einzelperson bewirkten Kapitaleinlage 
oder Reinvestition, so ist für die Rückführung dieses höheren Betrages die vorherige Genehmigung des SKKI einzuholen.

Art. 89: Ausländer, die in Vietnam im Rahmen eines Unternehmens-Kooperations-Vertrages oder bei einem Unternehmen mit ausländischem Investitionskapital beschäftigt sind, haben das Recht, ihre Löhne, Gehälter und andere rechtmäßig erworbene Einkünfte nach Abzug der Einkommensteuer und Lebenshaltungskosten für Vietnam in ausländischer Währung ins Ausland zu transferieren.

Art. 90: Die Konvertierung ausländischer in vietnamesische Währung und umgekehrt zu Zwecken der Investition, der Repatriienung oder des Transfers von Kapital ins Ausland oder der Geschäftstätigkeit des Unternehmens erfolgt nach dem von der Staatsbank von Vietnam bekanntgegebenen offiziellen Wechselkurs und in Übereinstimmung mit den geltenden Devisenkontrollvorschriften Vietnams.

Art. 91: Alle Transaktionen in ausländischer und vietnamesischer Währung sind ausschließlich über die in Artikel 85 dieses Dekrets genannten Banken vorzunehmen.

\section{Kapitel IX: Buchführung und Rechnungsprüfung}

Art. 92: Ein Unternehmen mit ausländischem Investitionskapital hat ordnungsgemäße Kontenbücher im Hinblick auf folgende Angelegenheiten zu führen:

(a) Vermögen, Materialien und Kapital, die für die Produktion und den Geschäftsbetrieb des Untemehmens verwendet werden, und deren Ein- und Ausgang;

(b) Einnahmen und Ausgaben;

(c) Verlauf und Ergebnis der Geschäfte des Unternehmens, einschließlich Käufe, Produktion, Absatz, Gewinne und Verluste und Verteilung der Einkünfte aus Produktion und Geschäftsbetrieb.

Der ausländische Partner einer vertraglichen Unternehmens-Kooperation hat eine dem Typ der Unternehmens-Kooperation entsprechende Buchhaltung zu führen.

Kontenbücher sind grundsätzlich in vietnamesischer Sprache zu führen. Aufgrund einer entsprechenden Vereinbarung zwischen den Joint-Venture-Partnem und nach Genehmigung durch das SKKI können sie jedoch in einer ausländischen Sprache geführt werden.

Art. 93: Das Untemehmen hat sicherzustellen, daß seine Buchführung ordnungsgemäß, angemessen, aktuell und systematisch ist. 
Art. 94: 1. Standardmaßeinheiten für Buchführungszwecke sind die geltenden offiziellen Maßeinheiten Vietnams. Alle anderen Maßeinheiten, die in Buchführungsdokumenten verwendet werden, müssen in die vietnamesischen offiziellen Maßeinheiten übertragen werden.

2. Grundsätzlich ist die Währungseinheit für Zwecke der Buchführung der vietnamesische Dong. Haben sich die beiden Partner auf eine ausländische Währung geeinigt und liegt dafür eine Genehmigung des SKKI vor, so kann auch eine ausländische Währung verwendet werden.

Art. 95: Das Finanzjahr eines Untemehmens mit ausländischem Investitionskapital entspricht dem in Artikel 78 dieses Dekrets vorgeschriebenen Steuerjahr.

Art. 96: Ein Untemehmen mit ausländischem Investitionskapital hat seine Kontenbücher in Übereinstimmung mit den allgemein anerkannten internationalen Buchführungsgrundsätzen und den vom Finanzministerium Vietnams anerkannten Standards zu führen.

Das Buchführungssystem umfaßt:

(a) das Kontensystem;

(b) die Form der Buchführung; und

(c) die Standards für Angaben in den Kontenbüchern.

Art. 97: 1. Die Kontenangaben müssen enthalten:
(a) die Bilanz;
(b) die Gewinn- und Verlustkonten; und
(c) den Bericht des Verwaltungsrates.

2. In der Bilanz sind Positionen, Quellen und Verwendung des Betriebskapitals am Ende eines Finanzjahres wiederzugeben.

Die Gewinn- und Verlustkonten müssen einen klaren Überblick über begründete Ausgaben und Einnahmẹn während des Finanzjahres und die Gewinne und Verluste des Unternehmens geben.

Der Bericht des Verwaltungsrates hat den wahren Stand der Geschäfte des Untermehmens, die Ergebnisse seiner Geschäfte und die Verteilung und Verwendung der aus Produktion und Geschäftstätigkeit erzielten Einkünfte anzugeben.

3. Die Angaben in der Rechnungslegung eines Unternehmens mit ausländischem Investitionskapital sind dem SKKI und den dem Finanzministerium unterstehenden Einkommensteuerbehörden innerhalb von drei Monaten nach Abschluß des Finanzjahres mitzuteilen. 
Art. 98: 1. Die Buchprüfung gegenüber den Untermehmen mit ausländischem Investitionskapital ist einmal jährlich zu einem festgelegten Zeitpunkt durchzuführen.

2. Das Finanzministerium Vietnams errichtet ein besonderes Gremium zur Prüfung der Buchführung und der Kontenangaben und zur Kommentierung des Buchführungssystems des Unternehmens.

Art. 99: Das Rechnungsprüfungsgremium hat einen Bericht über das Ergebnis seiner Tätigkeit folgenden Inhalts anzufertigen:

(1) Buchführung des Unternehmens;

(2) Ordnungsgemäßheit der Zahlen und der Kontenangaben;

(3) Beachtung des Systems und der Regelungen der Buchführung;

(4) Anwendung des Buchprüfungssystems durch das Unternehmen;

(5) Eingaben.

Art. 100: Der Rechnungsprüfungsbericht ist vor Ablauf der in Artikel 97 dieses Dekrets angeführten Frist für die Mitteilung über die Rechnungslegung abzuschließen und den zuständigen Behörden zuzuleiten.

\section{Kapitel X: Zoll, Einreise, Aufenthalt und Verkehr}

Art. 101: Unternehmen mit ausländischem Investitionskapital und Partner einer vertraglichen Unternehmens-Kooperation haben Einfuhrzölle auf Handelswaren der in Artikel 81 und 82 dieses Dekrets beschriebenen Art zu entrichten.

Art. 102: Persönliches Vermögen, das ein ausländischer Partner eines Unternehmens mit ausländischem Investitionskapital oder einer vertraglichen Untemehmens-Kooperation oder ein bei diesen Unternehmen beschäftigter Ausländer einführt, unterliegt in Übereinstimmung mit den geltenden Regelungen einer Vorzugsbehandlung.

Art. 103: Export- und Importlizenzen für Handelswaren erteilt das MfAW.

Lizenzen für Import und Export von persönlichem Vermögen der in Artikel 102 dieses Dekrets angeführten Art erteilt das Hauptzollamt.

Art. 104: Das Hauptzollamt schafft erleichterte Voraussetzungen für Zollerklärungen von Unternehmen mit ausländischem Investitionskapital und der dort beschäftigten Ausländer und von ausländischen Partnern vertraglicher Unternehmens-Kooperationen. 
Art. 105: Ausländern, die in Vietnam einreisen, um wegen eventueller Investitionen Untersuchungen durchzuführen und Vorbereitungen zu treffen, kann ein mehrfaches Einreisevisum für die Dauer von nicht mehr als drei Monaten erteilt werden. Dieses Visum kann um jeweils drei Monate verlängert werden.

Art. 106: Ausländern, die an der Durchführung eines Investitionsprojekts teilnehmen, einschließlich ihrer persönlichen Gehilfen, ist ein mehrfaches Einreisevisum für die Dauer von nicht mehr als einem Jahr zu erteilen, es kann jedoch unter Berücksichtigung der Laufzeit des Vertrages oder der Dauer der Auflösung eines Unternehmens oder der Beendigung des Vertrages mehrmals um jeweils ein Jahr verlängert werden.

Art. 107: 1. In der Regel werden Einreisevisa den betreffenden Personen spätestens innerhalb von fünf Monaten nach Erfüllung der Formalitäten der Antragstellung für Visa von den diplomatischen oder konsularischen Missionen der SR Vietnam in den ausländischen Staaten erteilt.

2. Ist der ausländische Antragsteller Bürger eines Staates, mit dem die Regierung der SR Vietnam eine Vereinbarung getroffen hat, wonach eine Ausnahmeregelung für Einreiseund Ausreisevisa gilt, geht diese Vereinbarung vor.

3. In dringenden Fällen, die eine sofortige Behebung eines unvorhersehbaren Notstandes oder eine technische Unterstützung für ein laufendes Investitionsprojekt erfordem, kann einem Ausländer unabhängig davon, ob er Mitglied des Unternehmens mit ausländischem Investitionskapital ist oder nicht, am Einreiseort ein Visum erteilt werden, vorausgesetzt, ein entsprechender Antrag ist von einem bevollmächtigten Vertreter des betreffenden Unternehmens oder von der vietnamesischen Wirtschaftsorganisation als Partner einer vertraglichen Unternehmens-Kooperation gestellt worden. Dieser Antrag ist vierundzwanzig Stunden vor der beabsichtigten Einreise beim SKKI zu stellen.

Art. 108: Jeder der in Artikel 105 und 106 dieses Dekrets genannten Ausländer genießt Freizügigkeit in den Gebieten, die für die Produktion und die Geschäftstätigkeit vorgesehen sind. Abgesehen von Sperrgebieten sind die betreffenden Gebiete beim SKKI registrieren zu lassen. Ein Ausländer kann auch in andere Gebiete reisen, wenn er dazu von den zuständigen Behörden die Erlaubnis erhalten hat. Sonstige touristische Aktivitäten unterliegen im übrigen der Regelung durch das Generalbüro für Tourismus.

Art. 109: Die in den vorstehenden Artikeln genannten Bestimmungen und Vorrechte betreffend Einreise, Aufenthalt und Freizügigkeit gelten auch für den Ehegatten, die Kinder und mitreisenden Familienangehörigen, einschließlich der in Artikel 106 erwähnten Haushaltshilfen des Ausländers während seines Aufenthaltes in Vietnam. 
Art. 110: Nach Erfüllung der erforderlichen Formalitäten gegenüber den zuständigen Postverwaltungsbehörden hat ein bei einem Unternehmen mit ausländischem Investitionskapital beschäftigter Ausländer folgende Rechte:

Bevorzugung bei der Benutzung der verfügbaren vietnamesischen Post- und Telekommunikationseinrichtungen für inländische und ausländische Kommunikation;

Errichtung eines eigenen Kommunikationssystems innerhalb des Unternehmens für Zwecke der Produktions- und Geschäftstätigkeit.

\section{Kapitel XI: Schlußbestimmungen}

Art. 111: Das vorliegende Dekret tritt am Tage seiner Ausfertigung in Kraft.

Art. 112: Der Minister für Außenwirtschaftsbeziehungen, der Minister für auswärtige Angelegenheiten, der Minister für Finanzen, der Minister für Arbeit, Kriegsversehrte und Soziales, der Minister des Inneren und der Generaldirektor der Hauptzollverwaltung erlassen innerhalb von spätestens fünfundvierzig Tagen vom Inkrafttreten dieses Dekrets an für ihren jeweiligen Zuständigkeitsbereich Rundschreiben betreffend die Durchführung dieses Dekrets.

Art. 113: Den Ministem, Präsidenten der Staatskomitees, Chefs der Abteilungen beim Ministerrat und Vorsitzenden der Volkskomitees der Provinzen, Städte und Sonderzonen unter Zentralverwaltung obliegt die Durchfühiung dieses Dekrets.

(Aus dem Englischen übersetzt: Arno Wohlgemuth) 